\title{
Physical aging and relaxation of residual stresses in a colloidal glass following flow cessation
}

\author{
Ajay Singh Nega and Chinedum O. Osujb) \\ Department of Chemical Engineering, Yale University, New Haven CT 06511
}

(Dated: 23 May 2018)

Dilute Laponite suspensions in water at low salt concentration form repulsive colloidal glasses which display physical aging. This phenomenon is still not completely understood and in particular, little is known about the connection between the flow history, as a determinant of the initial state of the system, and the subsequent aging dynamics. Using a stress controlled rheometer, we perform stress jump experiments to observe the elastic component of the flow stress that remains on cessation of flow or flow quenching. We investigate the connection between the dynamics of these residual stresses and the rate of physical aging upon quenching from different points on the steady state flow curve. Quenching from high rates produces a fluid state, $G^{\prime \prime}>G^{\prime}$, with small, fast relaxing residual stresses and rapid, sigmoidal aging of the complex modulus. Conversely, quenching from lower shear rates produces increasingly jammed states featuring slowly relaxing stresses and a slow increase of the complex modulus with system age. Flow cessation from a fixed shear rate with varying quench durations shows that slower quenches produce smaller residual stresses at short times which relax at long times by smaller extents, by comparison with faster quenches. These smaller stresses are correlated with a higher modulus but slower physical aging of the system. The characteristic time for the residual stress relaxation scales inversely with the quench rate. This implies a frustrated approach to any ideal stress-free state that succinctly reflects the frustrated nature of these glassy systems.

\footnotetext{
a) ajay.negi@yale.edu

b) chinedum.osuji@yale.edu
} 


\section{INTRODUCTION}

Many complex fluids show some degree of time dependence in their properties. Such displays of thixotropy are well known in colloidal dispersions for example, both under attractive and repulsive inter-particle potentials [Mewis (1979); Barnes (1997); Dullaert and Mewis $(2005 \mathrm{~b})]$. When brought to rest, the properties of these systems may continue to evolve as thermally driven particle motion drives structural rearrangements [Willenbacher (1996); Cipelletti et al. (2000); Abou et al. (2001); Suarez et al. (2009); Negi and Osuji (2009b)], a process known as physical aging. These rearrangements lead to slowly increasing viscosity and complex modulus as the system gradually "relaxes" and approaches some long time equilibrium or steady state at rest. There is qualitative similarity between the aging behavior of these colloidal systems and the glassy dynamics observed in polymer and molecular structural glasses, key among them the display of time-elapsed time rescaling of the dynamic properties [Cloitre et al. (2000); Cipelletti and Ramos (2002); Cloitre et al. (2003); Joshi and Reddy (2008)]. However, non-trivial distinctions still separate the behavior of colloidal systems from that of traditional glasses, as highlighted recently [McKenna et al. (2009)]. The viscosity of these fluids under flow is comprised of both hydrodynamic and elastic or thermodynamic contributions. In dilute dispersions of Brownian hard spheres, based on Einstein's viscosity relation, the elastic and viscous terms have been given by Batchelor Batchelor (1977)] in Equations 1,2

$$
\begin{aligned}
& \eta_{r}^{v}=1+2.5 \varphi+5.2 \varphi^{2} \\
& \eta_{r}^{e}=0.97 \varphi^{2}
\end{aligned}
$$

where $\eta_{r}^{v}$ is the viscous contribution to the relative viscosity, $\eta_{r}^{e}$ is the elastic (Brownian) contribution and $\varphi$ is the volume fraction of particles in the fluid. More recent models have extended these considerations into concentrated Brownian dispersions [Brady (1993)] as well as weakly aggregated systems [Potanin et al. (1995)]. In flocculating systems, the elastic term depends on the strength of inter-particle attractions due to the presence of load-bearing flocs, the size of which varies with the shear stress applied to the system Sonntag and Russel (1987a,b); Osuji et al. (2008)].

Deconvolution of the viscosity into rate dependent elastic and viscous terms can be made 
via stress jump measurements where a suspension in steady state flow is subjected to a sudden drop to zero shear rate, $\dot{\gamma}=0$. The viscous component of the shear stress dissipates nearly instantaneously, leaving only the elastic component as the measurable quantity that contributes to the viscosity decay function, $\eta_{-}(t, \dot{\gamma})$ [Watanabe et al. (1996)]. Suitable modification of strain controlled rheometers employing a force rebalancing transducer for torque measurements have enabled observations of the elastic stress component at times as early as $20 \mathrm{~ms}$ after cessation of flow Mackay et al. (1992); Kaffashi et al. (1997); Dullaert and Mewis (2005a)] . Extrapolation of the time dependence of the stress back to the cessation of shear at $t=0$ allows the steady state flow curve to be represented in terms of the rate dependent elastic and viscous contributions to the system viscosity as the shear stress before the jump is given as $\sigma(\dot{\gamma})=\sigma^{v}+\sigma^{e}=\left(\eta^{v}+\eta^{e}\right) \dot{\gamma}$. The difficulty of performing these measurements is matched by the insight they provide into the dispersion rheology. For example, in flocculating materials, the absence of hydrocluster induced shear thickening has now been attributed to the precipitous decrease in the elastic component of the shear stress with increasing shear rate, compared to the rather more modest increase of the hydrodynamic component [Gopalakrishnan and Zukoski (2004)].

In addition to decoupling the flow stress components, stress jump measurements also permit the observation of the relaxation of the elastic stress. A single exponential form was observed in weakly flocculated colloidal dispersions of fumed silica and carbon black [Dullaert and Mewis (2005a)] out to $\approx 0.1 \mathrm{~s}$, whereas a power-law dependence was found in hard sphere systems [Kaffashi et al. (1997); O'Brien and Mackay (2000)] out to $\approx 1$ s. Stress jump measurements have also been applied in polymer systems to follow the relaxation of deformed interfaces in immiscible blends of poly(isobutylene) in poly(dimethylsiloxane) (PDMS), where an exponential dependence was found, out to $4 \mathrm{~s}$ [Vinckier et al. (1997)]. In more recent work on polymer-clay nanocomposites, single exponential relaxations of the elastic flow stress component were observed, both for a Newtonian suspending polymeric fluid [Mobuchon et al. (2007)] as well as for a low molar mass PDMS medium [Zhu et al. $(2009)$ ], both out to $\approx 0.2 \mathrm{~s}$. Clearly, the dynamics of the elastic stress displayed on cessation of shear, the residual stress, so to speak, can provide important insights into the microscopics of the system under study. In materials which display physical aging, the same thermally driven structural rearrangements which give rise to increasing moduli are also responsible for the decay of the residual stress. Thus, one should expect that the time evolution of 
the system properties should be related in some way to the dynamics of this stress relaxation. Surprisingly, this connection remains quite unexplored and residual stress dynamics in colloidal systems have typically been considered only at short times scales, versus the comparatively long times at which physical aging is studied.

Here, we perform stress jump measurements on a Laponite dispersion and monitor the relaxation of the residual stress out to $100 \mathrm{~s}$, as a function of the rate of flow arrest. We also study the physical aging of the system via small amplitude strain controlled dynamic tests following cessation of shear. Our data reveal striking parallels between the physical aging and residual stress relaxation dynamics. In particular, the characteristic time for the stress relaxation increases with the duration of the flow quench step. Concurrently, the rate of physical aging also decreases, implying a frustrated approach to the long time equilibrium state of the system.

\section{EXPERIMENT}

\section{A. Materials}

Our system is an aqueous dispersion of Laponite which consists of disc-like clay particles of roughly $25 \mathrm{~nm}$ diameter and $1 \mathrm{~nm}$ thickness. Repulsive glasses are formed at low ionic strength in fairly dilute conditions due to long range electrostatic repulsion between the particles Bonn et al. (1998). The system displays glassy dynamics, as studied by scattering Knaebel et al. (2000); Abou et al. (2001); Bandyopadhyav et al. (2004)] and rheological means [Bonn et al. (2002); Abou et al. (2003); Joshi and Reddy (2008)]. Samples of concentrations $\varphi=3,4$ and 5 wt.\% are prepared by dispersion of Laponite XLG powder (Southern Clay Products) into ultra-pure water, adjusted to $\mathrm{pH} 9.5$ via addition of $\mathrm{NaOH}$ to ensure chemical stability of the particles [Mourchid and Levitz (1998)]. The samples are agitated vigorously for 20 minutes and then allowed to develop completely for 5 days under quiescent conditions, defining a well controlled and reproducible initial state.

\section{B. Method}

Rheology is conducted in strain controlled mode using an MCR301 instrument (AntonPaar) in the cone-plate geometry $\left(1^{\circ}, 50 \mathrm{~mm}\right.$ diameter steel cone). Evaporation of water 
from the sample was successfully suppressed without perturbing our system via application of a thin film of mineral oil at the sample edge. Steady state flow curves were collected from $\dot{\gamma}=10^{-2}$ to $10^{4} \mathrm{~s}^{-1}$ using a logarithmically varying equilibration time from $60 \mathrm{~s}$ at the lowest shear rates to $1 \mathrm{~s}$ at the highest shear rates. Conventional stress relaxation data were taken after a resting period of $300 \mathrm{~s}$ following a pre-shear at $100 \mathrm{~s}^{-1}$, using a strain of $\gamma=3 \%$, which is within the linear viscoelastic regime for all compositions studied. Frequency sweeps were carried out using this same strain from 100 to $1 \mathrm{rad} / \mathrm{s}$.

\section{Variation of pre-shear rate}

Samples were subjected to varying pre-shear flows at rates between 4 and $1000 s^{-1}$ for a duration of $100 \mathrm{~s}$. In each case, this time was more than sufficient to establish a robust steady-state in which the viscosity of the sample was well defined within $\pm 5 \%$. The samples were subjected to a linear quench of $1 \mathrm{~s}$ duration $\left(t_{q}=1 \mathrm{~s}\right)$ from the flowing state to the stationary or at-rest state. Physical aging and stress relaxation measurements were carried out on the system following separate pre-shear and mechanical quenching steps as just described. The physical aging of the samples after cessation of flow was monitored via the time evolution of the complex shear modulus in the linear viscoelastic regime using strain controlled oscillations at $\gamma=3 \%$ at an angular frequency of $\omega=10 \mathrm{rad} / \mathrm{s}$. The relaxation of the residual elastic component of the flow stress in the system after cessation of flow, here termed the residual stress, was then characterized, as described in a prior publication

[Negi and Osuji (2009c)]. Briefly, a constant zero strain rate condition was imposed on the sample. The stress required to maintain this $\dot{\gamma}=0$ stationary condition is the residual stress. Checks were performed using a Newtonian fluid, which should have no measurable residual stress, to verify the ability of the instrument to accurately make the measurements reported here.

\section{Variation of quench duration}

Samples were pre-sheared at a fixed rate of $\dot{\gamma}=100 \mathrm{~s}^{-1}$ for $100 \mathrm{~s}$ and then brought to rest via a linear ramp over varying durations, $t_{q}$, from 1 to $300 \mathrm{~s}$. Following this cessation of flow, physical aging was monitored as above via the time evolution of the complex modulus 
in the linear viscoelastic regime, $\gamma=3.0 \%$ and $\omega=10 \mathrm{rad} / \mathrm{s}$. Following the same pre-shear and flow cessation, the relaxation of residual stresses were monitored, as described above.

\section{RESULTS AND DISCUSSION}

\section{A. Effects of Pre-Shear Rate}

Laponite forms a yield stress fluid when dispersed in water, even at relatively low concentrations. As a result, the steady flow curves do not display Newtonian plateaus at low shear rates, as shown in Figure 1. The fluids display monotonic shear thinning, with shear thinning exponents $|n|>0.9$ for all three concentrations considered here. We do not observe high shear rate viscosity plateaus, even out to $10^{4} \mathrm{~s}^{-1}$. This strong shear thinning behavior over a wide range of shear rates is typical of Laponite suspensions [Bonn et al. (2002); Abou et al. (2003)]. Additionally, we do not observe any stress plateaus in the flow curves, suggesting that there are no obvious intervening regimes of shear banding or other flow-induced structuring. Absence of such makes these suspensions ideal candidates for studies as conducted here.

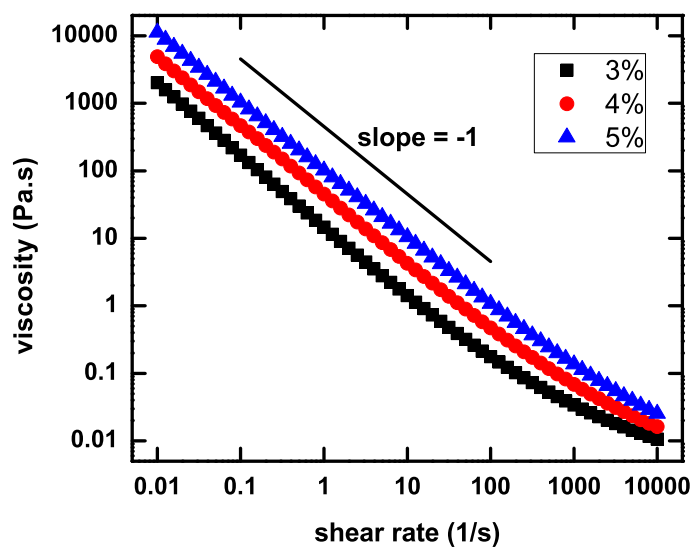

FIG. 1. Flow curves for 3, 4 and 5 wt.\% samples on decreasing shear rate.

Stress relaxation measurements on cessation of flow were performed for shear rates between 4 and $1000 \mathrm{~s}^{-1}$, as shown in Figure 2, The data show a slowing rate of decay of the residual stress as the initial shear rate of the system is decreased. In some cases, at longer times, there are slight increases in the stress which are attributed to structuring of the system at rest, which causes the viscosity of the suspension to rise with time. Because 
the rheometer in practice cannot maintain an identically zero shear rate (in general the minimum controllable was $\dot{\gamma} \approx 10^{-6} \mathrm{~s}^{-1}$ ), this results in an increasing component of the stress that may become measurable at long times. The data for 3 wt. $\%$ following cessation from $\dot{\gamma}=300 \mathrm{~s}^{-1}$ are particularly egregious however, for reasons that remain unclear. The instantaneous value of the residual stress immediately upon flow cessation is required to accurately determine the elastic component of the flowing stress at different points on the flow curve. Our measurements, however, did not permit observation of the residual stress at times shorter than $0.1 \mathrm{~s}$. We take $\sigma_{r}(t=0.2 \mathrm{~s})$ as representative of the elastic stress component, $\sigma^{e}$, during the immediately preceding flow, rather than performing a log-linear regression of the data to $t=0$, which, strictly speaking is required for this purpose. Performing a log-linear regression would imply the assumption that the same functional form is maintained at shorter times, which is something that we cannot justify with complete certainty. Despite the quantitative difference between the two estimates, the data do provide the same qualitative result. The elastic stress component can be expressed as a fraction of the overall flow stress as $R^{e}(\dot{\gamma})=\sigma_{r}(\dot{\gamma} ; t \approx 0) / \sigma(\dot{\gamma})$. As shown in Figure 3 , the elastic stress component decreases dramatically with increasing shear rate, illustrating the strong reduction in the cohesion of particle assemblies under flow. While this approach results in an underestimate of the elastic stress component, it does capture the large decrease in $R^{e}$ with increasing shear rate that is expected in these systems.

Physical aging measurements following rate quenches from the same flow rates as above show that at long times, all samples followed what appeared to be a weak power-law evolution of the complex modulus with time, Figure 4. However, the behavior at short times varied strongly with the initial shear rate, and the vertical separation at long times increased with increasing volume fraction. Quenches from the lowest shear rate, $\dot{\gamma}=4 \mathrm{~s}^{-1}$ produced a higher complex modulus, with $G^{\prime}>G^{\prime \prime}$, at short times which evolved with a monotonic near power law display throughout. By contrast, quenches from the highest shear rates $\dot{\gamma}=1000 \mathrm{~s}^{-1}$ produced systems that were initially fluid-like, with $G^{\prime \prime}>G^{\prime}$ at short times, for $\varphi=3$ wt.\%. The complex modulus evolved in a sigmoidal fashion, rapidly increasing at intermediate times before assuming the weak near power law growth at long times. Quenches from intermediate shear rates showed a smooth variation between these two limiting behaviors. No intersection of the different trajectories was observed during the measurement.

The sigmoidal variation of the modulus in time is quite striking, but not entirely unex- 

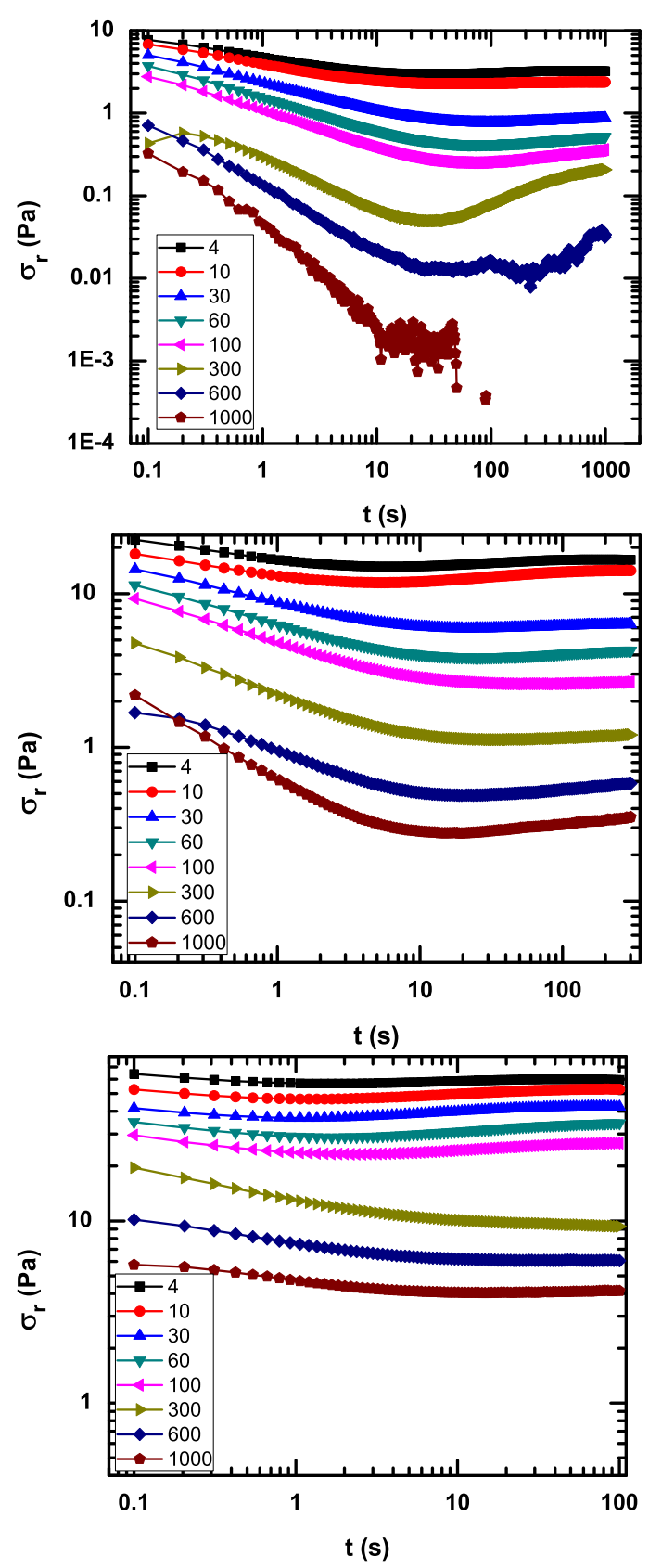

FIG. 2. Relaxation of residual stress or elastic stress component for 3, 4 and 5 wt.\% samples (top to bottom) following stress jump from different shear rates on the flow curve as indicated.

pected. While many disordered colloidal systems display power-law aging as implicit in timeelapsed time rescaling [Cloitre et al. (2000); Knaebel et al. (2000); Ramos and Cipelletti (2005)], intuitively, one understand that the power law dependence cannot persist indefinitely, if it is assumed that the system actually has a well defined, finite state or modulus at long time. Part of the difficulty in studying these systems, however, is the slow nature of 


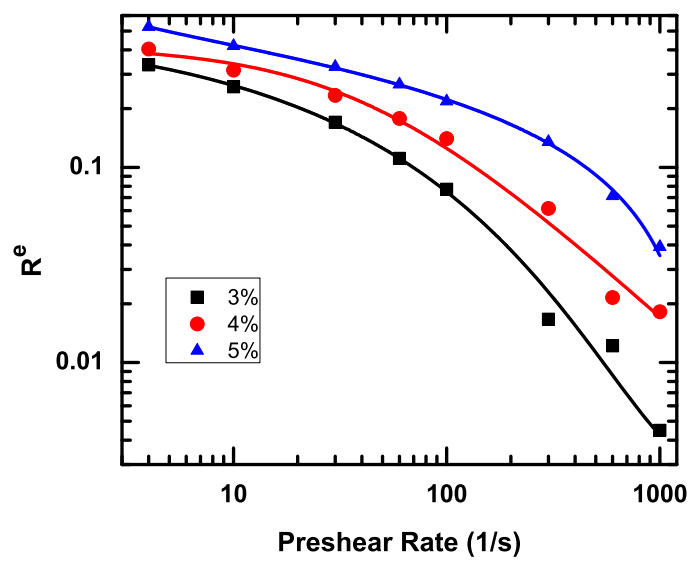

FIG. 3. Ratio of elastic stress at $t=0.2 \mathrm{~s}$ to the overall flow stress as a function of the shear rate. Lines are drawn as a guide to the eye.

the dynamics, and the slowing of the dynamics with the approach to equilibrium or steady state. Here we see that by starting from a highly fluid state characterized by a vanishing elastic stress component, we are able to access the short time regime of the system where it displays a sigmoidal response as it ages at first slowly, then rapidly, then slowly again as it approaches its long time steady state. This rapid evolution of the complex modulus is mirrored in the rapid relaxation of the residual stress for samples quenched from the highest shear rates. For a fixed shear rate, moving to higher concentrations increases the residual stress component and slows the dynamics of the system overall. A Carreau-like model, Equation 3 was used to estimate a characteristic relaxation time for the sigmoidal evolution of the complex modulus.

$$
G^{*}(t)=G^{*}(\infty)+\frac{G^{*}(0)-G^{*}(\infty)}{\left[1+(t / \tau)^{p}\right]}
$$

The rate of aging can be represented by the parameter $p$ which gives an indication of the steepness of the curve near the midpoint, $t=\tau$, of the growth between presumed constant $t=0$ and $t=\infty$ values. The inset plots of Figure 4 show the increase of $p$ with increasing shear rate before flow cessation, as described. Interestingly, a sigmoidal response has also been observed in the time evolution of the elastic modulus of a system composed of multi-arm star polymers in solution [Christopoulou et al. (2009)] The soft colloidal glass thus formed has important differences from the currently considered Laponite suspension. Notably, aging in the polymer glass proceeds via a complex two-step mechanism Helgeson et al. (2007); 

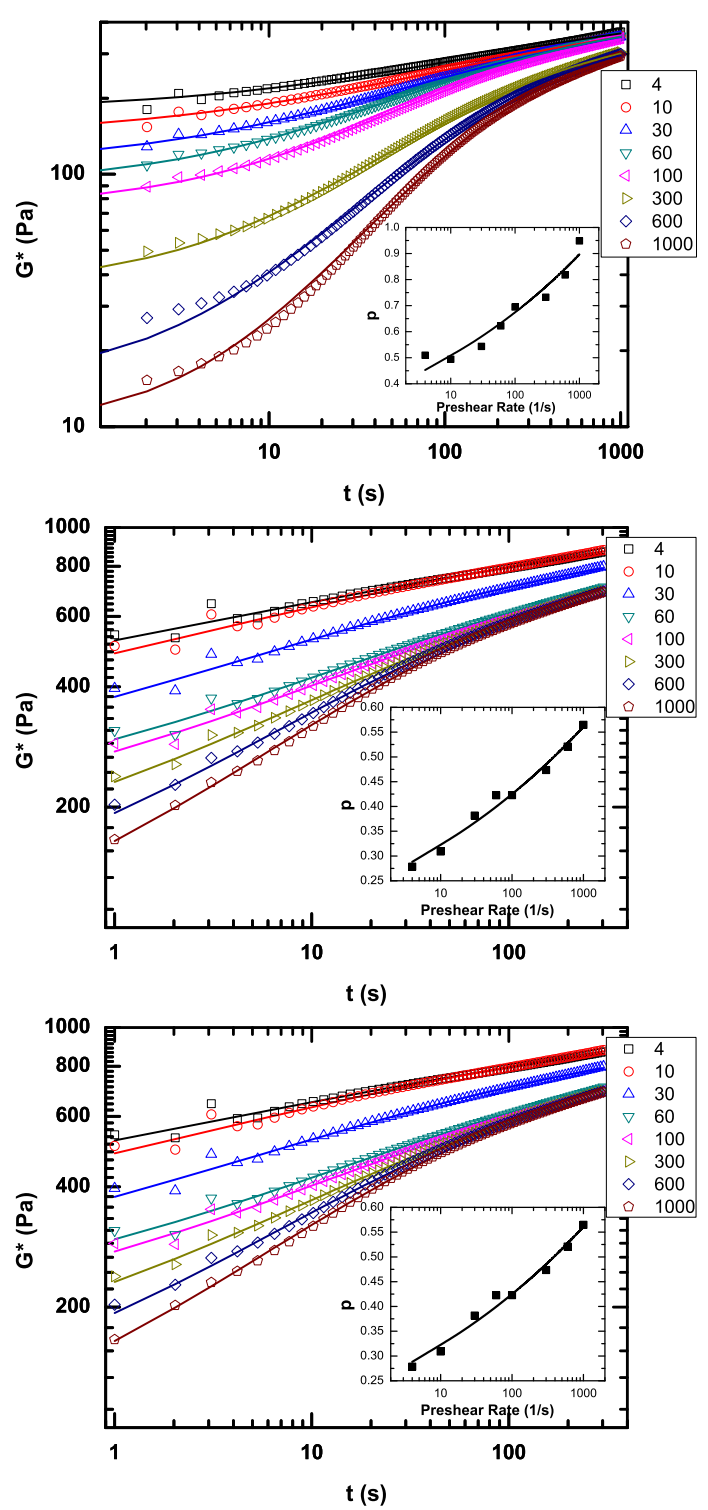

FIG. 4. Evolution of the complex modulus for 3, 4 and 5 wt.\% samples (top to bottom) following stress jumps from different shear rates on the flow curve as indicated. The lines are fits to the data using Equation 3, Oscillations were performed using a strain of $\gamma=3 \%$ at an angular frequency of $10 \mathrm{rad} / \mathrm{s}$. Inset: Value of the exponent $p$ in Equation 3 describing the slope of $G^{*}(t)$ in the vicinity of $t=\tau$. Lines are drawn as a guide to the eye.

Rogers et al. (2010)], and the system is able to access a presumed equilibrium state at long times $(\approx 5 \mathrm{E} 4 \mathrm{~s})$ where the modulus is stable in time. Nevertheless, the parallels are noteworthy.

The correlation between the earliest measured modulus of the system and the short time 
value of the residual stress is shown in Figure 5. Smaller residual stresses are produced by quenches from higher shear rates and are correlated to lower values of the complex modulus. A linear dependence has been observed between the elastic modulus and the residual stress in particulate dispersions with attractive interactions [Osuji et al. (2008); Ovarlez and Chateau (2008)]. Here, the dependence is roughly linear, although for reasons that remain unclear, the low stress data for $\varphi=4$ wt. $\%$ are somewhat non-conforming.

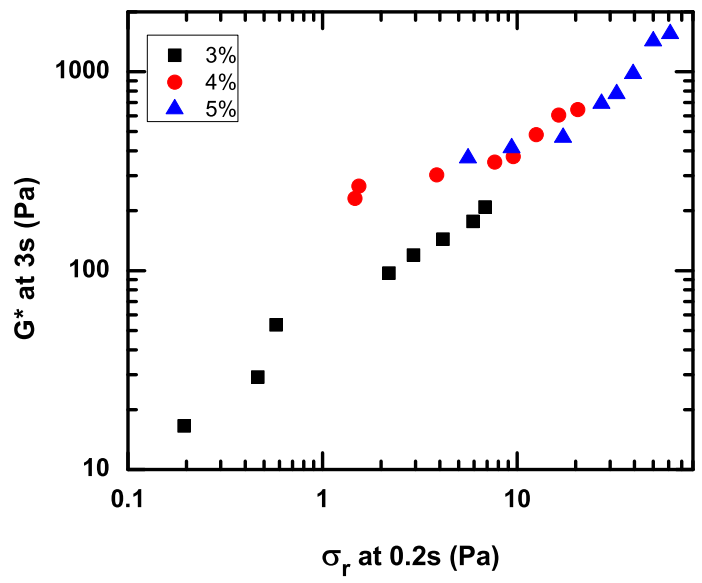

FIG. 5. Relation between the modulus and the stress on shear cessation, for different pre-shear rates.

\section{B. Effects of Quench Duration}

The preceding data clearly show that the dynamics of physical aging are paralleled by those of residual stress relaxation. This is not entirely surprising as the same thermally driven particle motion that gives rise to rearrangements that age the system also result in the relaxation of the elastic stresses built into the system on cessation of flow. Additionally, aging from a more fluid state should proceed faster than from a more jammed state. If we now consider quenches from a fixed shear rate, but over varying durations, we should expect to observe at some point the finite effects of a non-steady state flow history on the sample. A rapid flow cessation transitions the system from a free flowing steady state to a stationary, jammed state in a short time. Increasing the duration of the quench allows the system to sample configurations at lower shear rates en-route to the stationary state. The equilibrium condition at any particular shear rate is provided by the known viscosity from 
the steady state flow curve and so the instantaneous viscosity during the quench provides a good metric for the departure of the system from equilibrium during the cessation of flow. Over a range of quench durations, the system should display a spectrum of behavior in which the viscosity at any given shear rate during the quench deviates from the steady state value by an amount that scales with the quench rate. We monitor the instantaneous viscosity during the cessation of flow and find precisely this behavior, shown in Figure 6 . As expected, the magnitude of the shear thinning exponent, $n$ where $\eta(\dot{\gamma}) \sim \dot{\gamma}^{n}$ decreases continuously, in a linear fashion, as the quench duration is decreased.

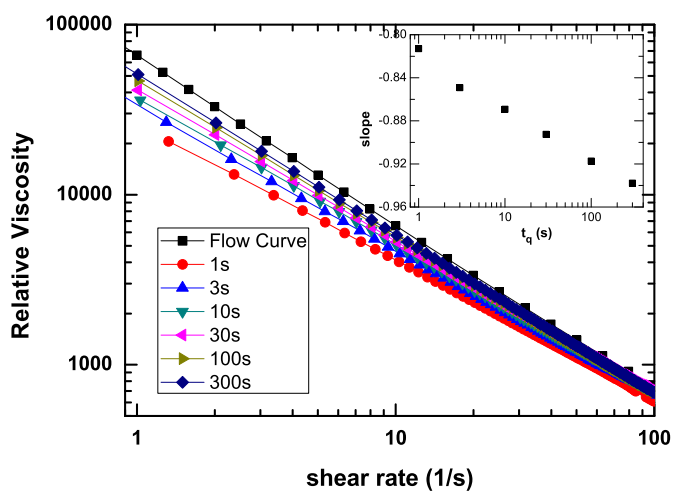

FIG. 6. Different quench rates result in the samples traversing different paths in the ergodic to non-ergodic transition on cessation of flow. Data are for a 4 wt.\% sample. Inset: Shear thinning exponent during the quench decreases in magnitude as a function of decreasing quench duration.

Prior to measurement of residual stresses, as well as for the modulus measurements, samples were subjected to a rejuvenating flow at the selected shear rate, $\dot{\gamma}=100 \mathrm{~s}^{-1}$. During these rejuvenating steps, the flow stress and thus the viscosity evolve to well defined levels that are characteristic of the steady state of the system at the applied flow rate (insets, Figure (7). The elastic stress that results on flow cessation is due to the structuring of the system that takes place during the finite quench interval. Surprisingly, we find that fast quenches lead to a higher residual stress than produced for slow quenches. Additionally, the residual stresses relax faster and to lower values for fast quenches than those produced by slower quenches, Figure 7. The data are strikingly different in form than those for a conventional stress relaxation, in response to a step-strain. They exhibit neither a power law nor stretched exponential dependence on time, but instead are suggestive of a sigmoidal approach to a finite, non-zero value at long times, much like the residual stress data of 
Figure 2. Notably, the relaxation shows a clear and monotonic dependence on the quench duration, $t_{q}$.
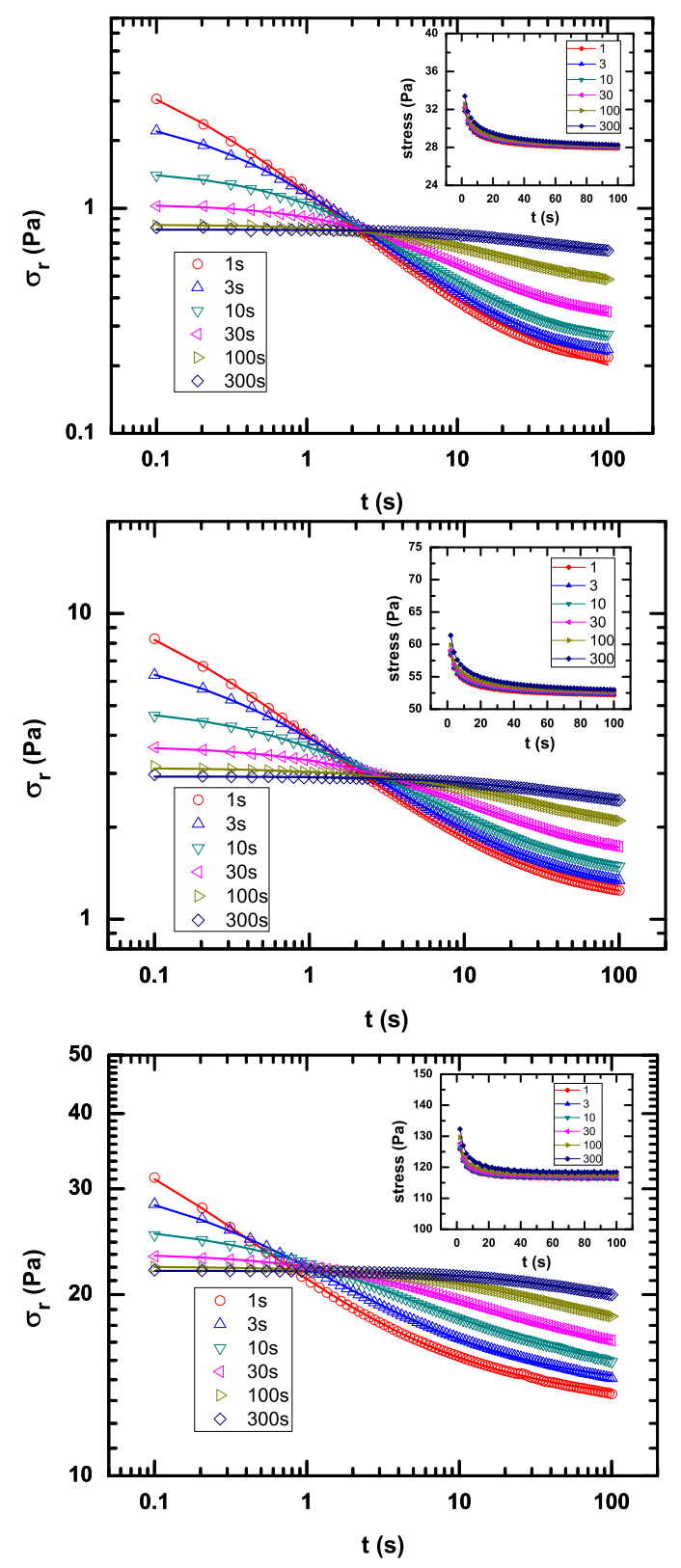

FIG. 7. Evolution of residual stress for different quench durations as indicated, for 3, 4 and 5 wt.\% samples (top to bottom) quenched from $100 \mathrm{~s}^{-1}$. Lines are fits to the data using Equation 4. The insets show the flow stress during the rejuvenation steps, indicating that the system starts from a consistent state.

We fit our data with good fidelity again using a sigmoidal function, Equation 4 for the time dependence of the residual stress, $\sigma_{r}(t)$. Here, again, $\tau$ is a characteristic time that 


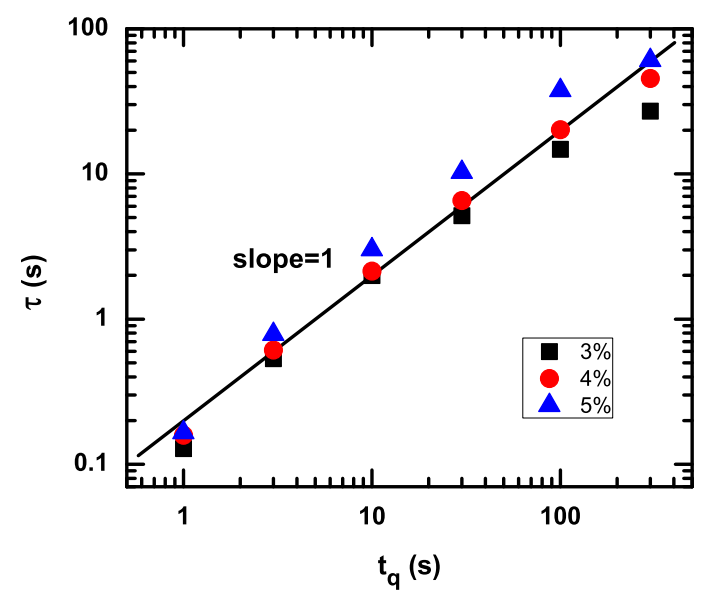

FIG. 8. Quench time dependence of the characteristic stress relaxation time, $\tau$ derived from Equation 4. The line shows a slope of 1.

describes the decrease of the sigmoidal function to its vertical midpoint between the value at $t=0, \sigma(0)$ and at $t=\infty, \sigma(\infty)$, and the exponent $p$ characterizes the slope of the function in the vicinity of $t=\tau$. Remarkably, we find that the characteristic time for the relaxation of the residual stress is a linear function of the quench time, $\tau \sim t_{q}$, as shown in Figure 8 .

$$
\sigma_{r}(t)=\sigma_{r}(\infty)+\frac{\sigma_{r}(0)-\sigma_{r}(\infty)}{\left[1+(t / \tau)^{p}\right]}
$$

The modulus of the glass, measured immediately after cessation of shear is inversely proportional to the rate of quench. The slowest quench rates, with flow cessation over periods of $300 \mathrm{~s}$, lead to the highest moduli. The modulus shows a slow increase that is well described by a power law at late times, but with significant sigmoidal character at shorter times, Figure 9. This is not unlike the response observed for quenches from different initial shear rates. Attempts to superpose the data by time-elapsed time rescaling fail, Figure 10. That is, shifting in time to account for the true sample age due to passage of time during the quench does not produce a satisfactory superposition of data. These results show that the flow history of the sample is important in determining its aging path, and different trajectories through the non-steady state flow curves en route to the stationary state invariably result in different and non-superposable aging behaviors thereafter. The correlation between modulus and residual stress is shown in Figure 11. Here now, there is no longer a simple direct correspondence between stresses and moduli. The residual stress displayed is not just function of structuring that occurs during the finite quench, but also 
relaxation of elastic components that were supported at the start of the quench.
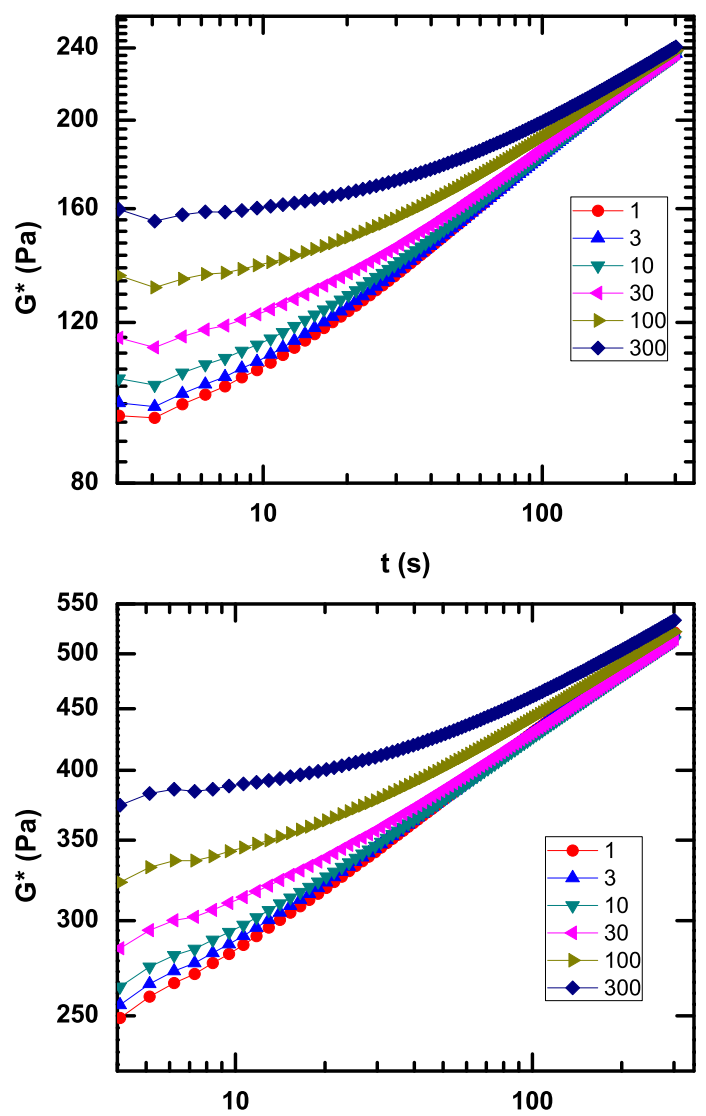

$t(s)$

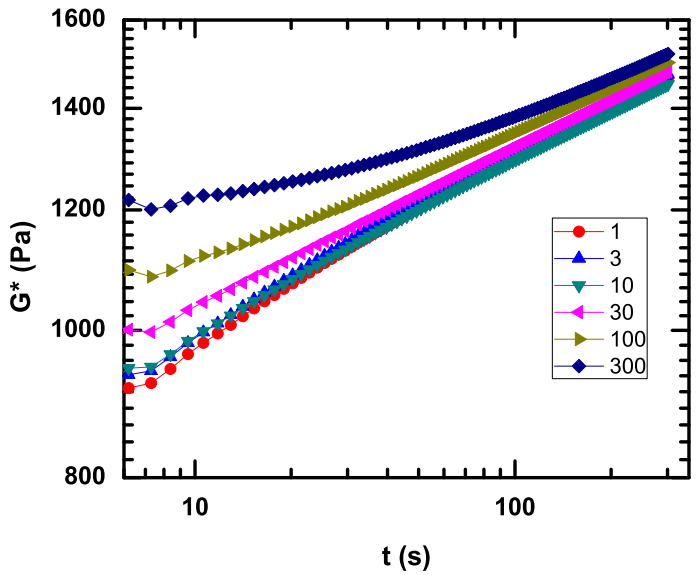

FIG. 9. Evolution of the complex modulus in 3, 4 and 5 wt.\% samples (top to bottom) quenched from $\dot{\gamma}=100 \mathrm{~s}^{-1}$. Oscillations are performed using a strain of $\gamma=3 \%$ at an angular frequency of $10 \mathrm{rad} / \mathrm{s}$. The legend indicates the duration of the quench, $t_{q}$, in seconds.

The data for the residual stress relaxation can be contrasted with that obtained by traditional step-strain experiments as well as frequency dependent moduli. Frequency sweeps of 


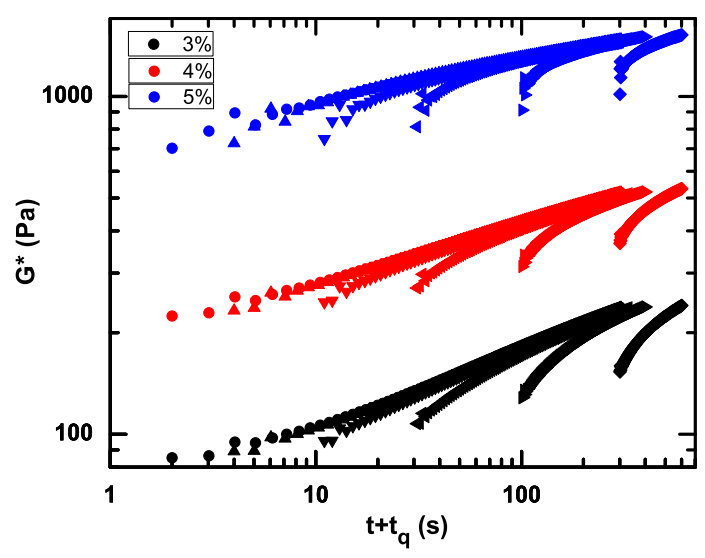

FIG. 10. Evolution of the complex modulus, shifted according to the quench durations.

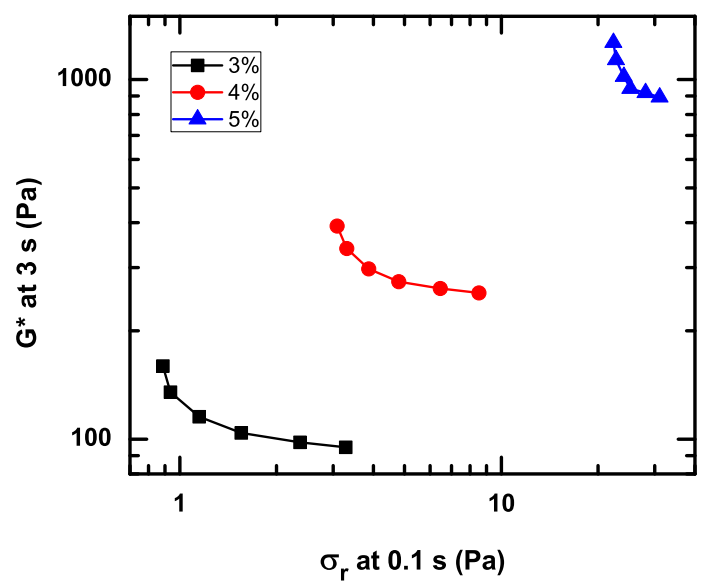

FIG. 11. Relation between the modulus and the stress on shear cessation, for different quench rates.

the suspensions show a very weak dependence of both storage and loss moduli on frequency, Figure 12, Data was collected after quenching from $\dot{\gamma}=100 \mathrm{~s}^{-1}$ over 1,10 and $100 \mathrm{~s}$, following which the samples were allowed to sit quiescently for $300 \mathrm{~s}$ prior to measurement. This ensured that the measurement time $(\approx 100 \mathrm{~s})$ was small enough compared to the sample age that changes in the system properties due to aging during the measurement were reasonably small. Similarly, step strain experiments were performed after an $300 \mathrm{~s}$ quiescent period to evaluate the conventional stress relaxation behavior. A strain of $\gamma=3 \%$ was used, which is in the linear regime for the system, as confirmed by strain sweep experiments. The data, Figure 13, are in good agreement with the slow relaxation that is inferred from the $G(\omega)$ data of Figure 12. Less than half a decade of relaxation is observed over 3 decades of time for 3 wt. $\%$, and substantially less than this for 4 and 5 wt.\%. For both $G(\omega)$ and $G(t)$, there 


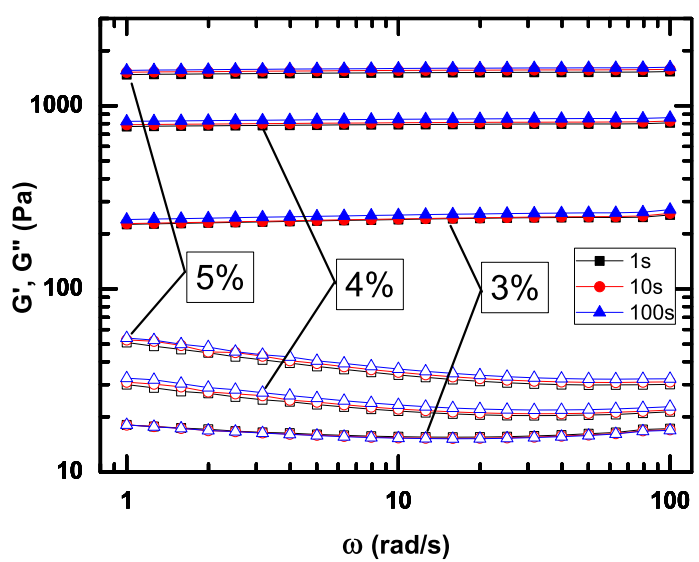

FIG. 12. Frequency sweep measurements. Samples were quenched over the 3 different times indicated and aged for $300 \mathrm{~s}$ before measurement.

is little dependence on the quench rate both in the absolute values of the moduli measured, and, more significantly, in their time(frequency) dependences.

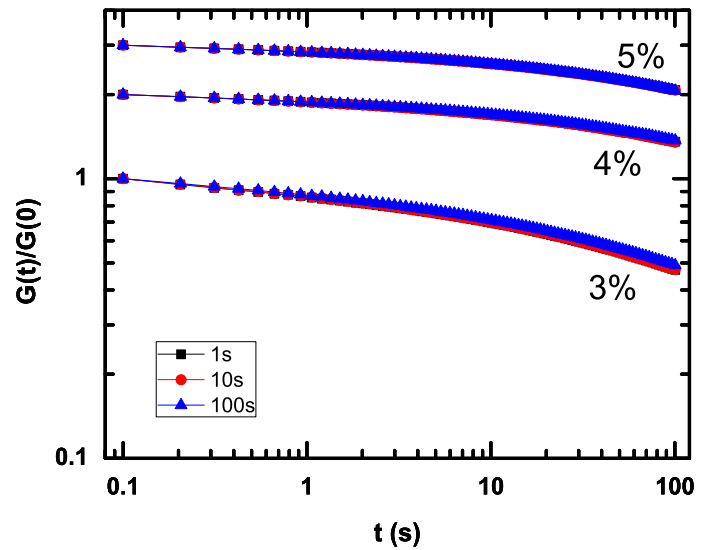

FIG. 13. Conventional stress relaxation measurements. Samples were quenched over the 3 different times indicated and aged for $300 \mathrm{~s}$ before measurement.

\section{CONCLUSION}

In summary, our investigation has shown that the quench rate, as well as the initial flow rate from which the system is quenched, both strongly influence the aging dynamics. The effects of the initial flow rate are particularly intuitive, assuming an infinitely fast quench such that the properties (structure) immediately on cessation of flow are those of the immediately preceding flow regime. One can readily imagine that a system that is in 
steady flow at an infinitely low shear rate, $\dot{\gamma} \rightarrow 0$, would display an infinitesimal rate of aging upon cessation of flow. Conversely, a system in steady flow at a very high shear rate, $\dot{\gamma} \rightarrow \infty$, is completely fluidized, and should display the the full aging response from fluid to semi-solid as the system undergoes structural arrest and further vitrifies with passage of time after cessation of flow. For all finite initial shear rates, the displayed behavior should fall on a spectrum between these two limiting cases, as described in Figure 14. Whether or not a power-law or logarithmic or even exponential aging response is observed then becomes a function of the initial flow rate of the system and the duration of the observation window, among other factors. Promisingly, recent work in this area has been successful in revealing both the stress [Negi and Osuij (2009a)] and frequency [Negi and Osuii (2010)] dependence of the complex modulus during structural arrest following rapid cessation of flow from high shear rates. The behavior observed for the currently studied Laponite system is in broad agreement with this framework of a globally sigmoidal response which we advance as a generic response for out of equilibrium materials. This argument has also been given based on earlier work in polymer glasses, where a sigmoidal form is suggested for a perfect thermal quench, with power-law aging simply the consequence of a limited observation or aging time window [McKenna (2003)]. Here, abrupt mechanical quenching of the flowing suspension permits access to a larger spectrum of the system response. The lack of superposition of the complex modulus with waiting time may reflect the different energy landscapes or distribution of relaxation mechanisms available to the system as a function of the initial flow rate and the fact that the evolution of the landscapes is not along a single trajectory, but depends on the initial condition from which the system is evolved.

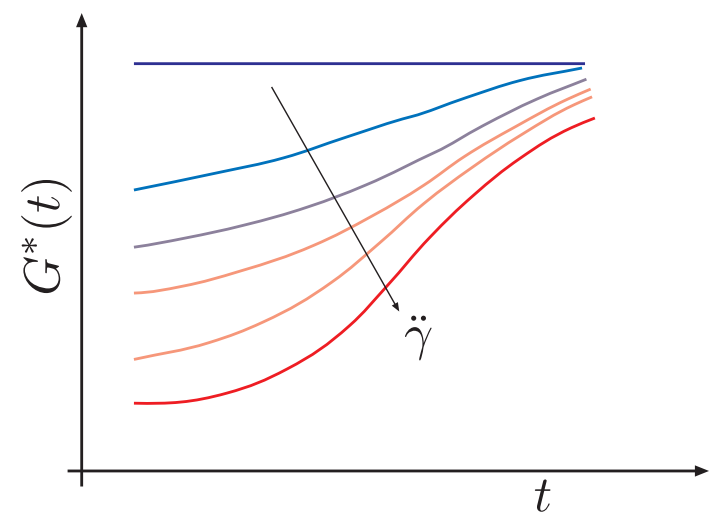

FIG. 14. Generic picture of the evolution of the complex modulus. 
With a fixed initial condition, the quench rate is seen to exert a strong influence on the aging dynamics in this repulsive colloidal glass and serves to characterize the departure of the system from equilibrium. Rapid quenches lead to faster aging from smaller moduli. Further, the aging dynamics are well correlated to the decay of residual stresses that are trapped in the glass at the ergodic to non-ergodic transition on cessation of flow. These stress dynamics are dependent on the quench rate and display a linear dependence of their characteristic relaxation time on the duration of the quench. Notably, they follow neither a simple exponential nor a power law form. The simultaneously decreasing initial value and final extent of relaxation of the residual stress concisely represent the frustrated and continuously slowing approach to equilibrium in these materials. This work suggests that the flow cessation rate may be identified as somewhat akin to an effective cooling rate for the colloidal glass wherein infinitely slow cooling, $t_{q} \rightarrow \infty$, maintains equilibrium and results in a stationary state on complete flow cessation. Many of the features displayed here resonate intuitively, and should be observed in other colloidal systems which display glassy or glass-like dynamics. Dynamic scattering can provide useful data in these systems and measurements which incorporate control over the flow arrest are a natural follow on to this work.

\section{ACKNOWLEDGMENTS}

The authors gratefully acknowledge NSF funding via CBET-0828905.

\section{REFERENCES}

Abou, B., Bonn, D., and Meunier, J., Phys. Rev. E 64, 021510 (2001).

Abou, B., Bonn, D., and Meunier, J., J. Rheol. 47, 979 (2003).

Bandyopadhyay, R., Liang, D., Yardimci, H., Sessoms, D. A., Borthwick, M. A., Mochrie, S. G. J., Harden, J. L., and Leheny, R. L., Phys. Rev. Lett. 93, 228302 (2004).

Barnes, H., J. Non-Newtonian Fluid Mech. 70, 1 (1997).

Batchelor, G. K., Journal of Fluid Mechanics 83, 97 (1977).

Bonn, D., Tanaka, H., Wegdam, G., Kellay, H., and Meunier, J., Europhys. Lett. 45, 52 (1998). 
Bonn, D., Tanase, S., Abou, B., Tanaka, H., and Meunier, J., Phys. Rev. Lett. 89, 015701 (2002).

Brady, J., Journal of Chemical Physics 99, 567 (1993).

Christopoulou, C., Petekidis, G., Erwin, B., Cloitre, M., and Vlassopoulos, D., Philosophical Transactions of the Royal Society A: Mathematical, Physical and Engineering Sciences 367, 5051 (2009).

Cipelletti, L., Manley, S., Ball, R., and Weitz, D., Phys. Rev. Lett. 84, 2275 (2000), ISSN 0031-9007.

Cipelletti, L. and Ramos, L., Curr. Opin. Colloid Interface Sci. 7, 228 (2002).

Cloitre, M., Borrega, R., and Leibler, L., Phys. Rev. Lett. 85, 4819 (2000).

Cloitre, M., Borrega, R., Monti, F., and Leibler, L., Phys. Rev. Lett. 90, 068303 (2003).

Dullaert, K. and Mewis, J., J. Colloid Interface Sci. 287, 542 (2005a).

Dullaert, K. and Mewis, J., Journal of Rheology 49, 1213 (2005b).

Gopalakrishnan, V. and Zukoski, C. F., J. Rheol. 48, 1321 (2004).

Helgeson, M. E., Wagner, N. J., and Vlassopoulos, D., J. Rheol. 51, 297 (2007).

Joshi, Y. M. and Reddy, G. R. K., Phys. Rev. E. 77, 021501 (2008).

Kaffashi, B., Obrien, V. T., Mackay, M. E., and Underwood, S. M., J. Colloid Interface Sci. 187, 22 (1997).

Knaebel, A., Bellour, M., Munch, J., Viasnoff, V., Lequeux, F., and Harden, J., Europhys. Lett. 52, 73 (2000).

Mackay, M., Liang, C., and Halley, P., Rheologica Acta 31, 481 (1992).

McKenna, G., J. Phys.: Condens. Matter 15, 737 (2003).

McKenna, G. B., Narita, T., and Lequeux, F., J. Rheol. 53, 489 (2009).

Mewis, J., Journal of Non-Newtonian Fluid Mechanics 6, 1 (1979).

Mobuchon, C., Carreau, P., and Heuzey, M., Rheologica Acta 46, 1045 (2007).

Mourchid, A. and Levitz, P., Phys. Rev. E 57, R4887 (1998).

Negi, A. S. and Osuji, C. O., arXiv arXiv:0910.1709v2 (2009a).

Negi, A. S. and Osuji, C. O., Phys. Rev. E. 80, 010404 (2009b).

Negi, A. S. and Osuji, C. O., Rheol. Acta 48 (2009c).

Negi, A. S. and Osuji, C. O., arXiv arXiv:1003.5203v1 (2010).

O'Brien, V. and Mackay, M., Langmuir 16, 7931 (2000).

Osuji, C. O., Kim, C., and Weitz, D. A., Phys. Rev. E. 77, 060402(R) (2008). 
Ovarlez, G. and Chateau, X., Phys. Rev. E. 77, 61403 (2008).

Potanin, A., Derooij, R., Van den Ende, D., and Mellema, J., Journal of Chemical Physics 102, $5845(1995)$.

Ramos, L. and Cipelletti, L., Phys. Rev. Lett. 94, 158301 (2005).

Rogers, S., Callaghan, P., Petekidis, G., and Vlassopoulos, D., Journal of Rheology 54, 133 (2010).

Sonntag, R. C. and Russel, W. B., J. Colloid Interface Sci. 113, 399 (1987a).

Sonntag, R. C. and Russel, W. B., J. Colloid Interface Sci. 115, 378 (1987b).

Suarez, M., Kern, N., Pitard, E., and Kob, W., The Journal of Chemical Physics 130, 194904 (2009).

Vinckier, I., Mewis, J., and Moldenaers, P., Rheologica Acta 36, 513 (1997).

Watanabe, H., Yao, M., Yamagishi, A., Osaki, K., Shitata, T., Niwa, H., and Morishima, Y., Rheologica Acta 35, 433 (1996).

Willenbacher, N., Journal of Colloid and Interface Science 182, 501 (1996).

Zhu, Y., Cardinaels, R., Mewis, J., and Moldenaers, P., Rheologica Acta 48, 1049 (2009). 